\title{
Light Scattering and SAXS Study of AOT Microemulsion at Low Size Droplet
}

\author{
Soheil Sharifi ${ }^{1}$, Masoud Amirkhani ${ }^{2}$, Jahanbakhsh Mashayekhi Asla ${ }^{1}$, Mohammad Reza Mohammadi ${ }^{1}$, \\ Othmar Marti ${ }^{2}$
}

${ }^{1}$ Department of Physics, University of Sistan and Baluchestan, Zahedan, Iran; ${ }^{2}$ Institut für Experimentelle Physik, Universität Ulm, Ulm, Germany.

Email: soheil.sharifi@gmail.com

Received February $5^{\text {th }}, 2011$; revised May $27^{\text {th }}, 2011$; accepted April $18^{\text {th }}, 2011$

\begin{abstract}
We study collective diffusion coefficient $\left(\mathrm{D}_{c}\right)$ of Water-in-oil nanoemulsions $\left(\mathrm{L}_{2}\right.$ phase $)$ stabilized by AOT and dispersed in n-Decane oils by dynamic light scattering (DLS). At constant water concentration we vary the oil concentration and there is clear evidence for a changing collective diffusion coefficient of the droplets in AOT nanoemulsion. The collective diffusion coefficient in AOT nanoemulsions is studied from relaxation investigations with dynamic light scattering. Also, we study the collective diffusion coefficient $\left(D_{c}\right)$ of droplets with add the TBAC to the droplets of AOT nanoemulsion. We discuss the results with study structural investigations with small-angle $\mathrm{x}$-ray scattering. The results of this study suggest that the formation of non-spherical aggregates at low concentration of droplets can describe the behavior of the collective diffusion coefficient at AOT nanoemulsion.
\end{abstract}

Keywords: Nano-Droplet; Microemulsions; Light Scattering; SAXS; Diffusion

\section{Introduction}

Brownian motion is a subject of renewed interest since the development of photon correlation spectroscopy (PCS) in the last decade. The dynamic properties of microemulsions and colloidal systems is studied by measuring the relaxation of concentration fluctuations, [1,2]. The relaxation time of the fluctuations can be expressed in a diffusion coefficient, which is called the collective diffusion coefficient $\left(D_{c}\right)$, in the limit of infinite dilution. The study of the collective diffusion coefficient of microemulsion is an interesting topic in soft matter physics. The microemulsions are thermodynamically stable mixtures of water, oil, and surfactant with nano-metric size of droplets to the solvents $\left(\mathrm{H}_{2} \mathrm{O}\right.$ or Oil). In the case of water/surfactants (droplet) to the nanopolar solvents (oil), microemulsions have $\mathrm{L}_{2}$ phase and oil/surfactant inside the water solutions is $\mathrm{L}_{1}$ phase of microemulsions. In our samples, the composition of each system is determined by the molar ratio $\mathrm{X}$ of water to surfactant molecules.

$$
\mathrm{X}=\frac{\left[\mathrm{H}_{2} \mathrm{O}\right]}{[\mathrm{AOT}]}
$$

and the droplet mass fraction (mf),

$$
\mathrm{mf}=\frac{\mathrm{m}_{\mathrm{AOT}}+\mathrm{m}_{\mathrm{H}_{2} \mathrm{O}}}{\mathrm{m}_{\mathrm{AOT}}+\mathrm{m}_{\mathrm{H}_{2} \mathrm{O}}+\mathrm{m}_{\mathrm{Dec}}}
$$

which varies by the respective mass of the components water $\left(\mathrm{m}_{\mathrm{H}_{2} \mathrm{O}}\right)$, decane $\left(\mathrm{m}_{\text {Dec }}\right)$, and AOT. In this work, the microemulsion system is located in the $\mathrm{L}_{2}$ region at studied temperatures and compositions investigated. Most works on collective diffusion coefficient of droplets and interactions of droplets have been done mainly on charged macromolecules, [3-15], for example bimolecular, [3-10], as DNA, proteins and amino acids, micelles, [11], and polymer latexes, [15], in water (as polar solvent) at low ionic strength. The strong, long range electrostatic interactions between the particles or droplets in these systems lead to remarkable structure effects. Only in the higher number densities or droplet concentration of microemulsions where the inter-particle separation is in the order of the particle dimensions hydrodynamic interactions can be observed.

In previous study of the collective diffusion coefficient in $\mathrm{C}_{12} \mathrm{E}_{5} / \mathrm{H}_{2} \mathrm{O} /$ Decane microemulsion, the collective diffusion coefficient as function of droplet mass fraction has positive slop at the low droplet mass fraction (dilute regime mass fraction below 0.1) that describes an attractive interaction between droplets. It is well known that $\mathrm{C}_{12} \mathrm{E}_{5} /-$ $\mathrm{H}_{2} \mathrm{O}$ /Decane microemulsion at constant surfactant-oil mass ratio of 1.08 with the different mass fractions has shown behaviors as the hard sphere droplets, [16-20]. The Collective diffusion coefficient as function of mass fraction in the $\mathrm{C}_{12} \mathrm{E}_{5} / \mathrm{H} 2 \mathrm{O} /$ Decane microemulsion has positive behavior and only depends to the inter-particle interaction of 
hard sphere droplet. The mixture of PEG with $\mathrm{C}_{12} \mathrm{E}_{5} / \mathrm{H}_{2} \mathrm{O} /-$ Decane microemulsion shown that addition of PEG causes slow down of the collective diffusion coefficient $D_{c}$. The measured $\mathrm{D}_{\mathrm{c}}$ data for the droplets in the presence of PEG are modeled using the Asakura-Oosawa theory of depletion, [20], but $\mathrm{D}_{\mathrm{c}}$ in the AOT/ $\mathrm{H}_{2} \mathrm{O} /$ Decane microemulsions has different behavior. Structure and phase behavior of AOT microemulsions are well investigated of a liquid of droplets on a nanometer scale, [21]. A study shown, for AOT $/ \mathrm{H}_{2} \mathrm{O} /$ Decane microemulsion, at the low droplet mass fractions $(0.01<\mathrm{mf}<0.1$ transition from dilute to semi-dilute regime) and molar ratio 40 the collective diffusion coefficient has a linear function of mass fraction. It is well known that at high water concentration with the molar ratio of $X=40$ the collective diffusion coefficient $\mathrm{D}_{\mathrm{c}}$ depends on the droplet mass fraction (droplet concentration) due to inter-particle interactions, [22]. This study is an attempt to understand the collective diffusion coefficient behavior of AOT $/ \mathrm{H}_{2} \mathrm{O} /$ Decane microemulsion at low water concentration (molar ratio, $\mathrm{X}=6.7$ ). The most study in this range of water concentration that shown droplet of the microemulsion has spherical behavior, $[23,24]$. In the present work, we studied the behavior of the collective diffusion coefficient at the molar ratio 6.7 and low mass fraction $(0.01<\mathrm{mf}<0.08)$, by means of DLS and SAXS techniques. Also, we study effect of TBAC on the $\mathrm{D}_{\mathrm{c}}$ in the $\mathrm{AOT} / \mathrm{H}_{2} \mathrm{O} /$ Decane microemulsion.

\section{Experimental}

\subsection{Methods and Materials}

Sodium-2-diethylhexyl sulfosuccinate, or AOT 99\% (an Alfa product), was dried in vacuum. Deionized and distilled water were employed to prepare the samples for the light scattering and SAXS measurements and solutions of the mix TBAC with microemulsions. The microemulsions were prepared by mixing of surfactants $\mathrm{AOT}, \mathrm{H}_{2} \mathrm{O}$, and oil (Decane) and waiting for several minutes until the samples were single phase and optically clear. Decane 99\% (Aldrich) and tetrabutylammonium chloride (TBAC) and n-decane were obtained from Sigma-Aldrich. The composition of the $A O T / \mathrm{H}_{2} \mathrm{O} /$ Decane microemulsion is given by the two parameters $\mathrm{X}$ and $\mathrm{mf}$. The mixing of TBAC with microemulsions is described with the molar ratio of TBAC to AOT, Equation (3).

$$
\mathrm{Y}=\frac{[\mathrm{TBAC}]}{[\mathrm{AOT}]}
$$

The experimental solutions were prepared at temperature $293.15 \mathrm{~K}$. The microemulsions were prepared by weighting appropriate amounts of AOT dissolving in decane then by adding water into the solution. Finally, all samples were filtered by using 0.2-pm Teflon filters (Gelman). The Small-angle X-ray scattering measurements were per- formed using the pinhole SAXS instrument at the University of Aarhus. The instrument consists of an X-ray camera (NanoSTAR, Bruker AXS) with a rotating anode X-ray $(\mathrm{Cu} \mathrm{K \alpha}$ radiation) source, cross-coupled Göbel mirrors, collimation using three pinholes and an evacuated beam path, and a $2 \mathrm{D}$ position-sensitive gas detector (HiSTAR). In the current experiments the small pinholes were used, giving a range of scattering vectors as 0.0084 $\leq \mathrm{Q}\left(\AA^{-1}\right) \leq 0.35$, where $\mathrm{Q}=(4 \pi / \lambda) \sin (\theta / 2)$ is the modulus of the wave vector, $\theta$ is the scattering angle, and $\lambda=1.542 \AA$ is the X-ray wavelength. The sample was held in a $1 \mathrm{~mm}$ glass capillary at room temperature. Data was acquired as a function of the scattering vector modulus $\mathrm{Q}=(4 \pi \cdot \sin \theta) / \lambda$ where $2 \theta$ is the angle between the incident and scattered photons. Dynamic light scattering measurements were performed on filtered samples using ALV CGS-8f/DLS Instruments Series A6160-V1052 at Ulm University. The scattering angle was maintained at $90^{\circ}$, and the temperature was kept at $293.15 \mathrm{~K}$ for the microemulsion samples.

\section{Results}

Microemulsions were formulated by mixing AOT with water and $n$-Decane at the fixed molar ratio of water to AOT $(\mathrm{X}=6.7)$ at the different mass fraction $(0.01<\mathrm{mf}<$ 0.07 dilute regime). The dynamic behavior of the AOT/$\mathrm{H}_{2} \mathrm{O} /$ Decane microemulsion was probed with dynamic light spectroscopy. The correlation function of the scattered light intensity showed a single exponential decay at all concentrations, Figure 1. (A). All the correlation functions in this work were fitted by a single stretched exponential function, [1,2].

$$
g_{1}(t)=\exp \left[-\left(\frac{t}{\tau}\right)^{\beta}\right]
$$

That $\tau=1 /\left(q^{2} D_{C}\right)$ and the collective diffusion coefficient $D_{c}$ was extracted as a function of the droplet mass fraction as shown in Figure 1(b). The collective diffusion coefficient measurements were carried out on dilution series of microemulsion samples at fixed temperature 293.15 $\mathrm{K}$ and molar ratio 6.7 with two different molar ratio of TBAC (0.0 and 0.049 molar/l). In the Figure 1, the collective diffusion coefficient show a liner behavior with negative slop between $0.01<\mathrm{mf}<0.07$. By increasing TBAC content relaxation time decreases, Figure 1(a) and the diffusion coefficient increases, Figure 1(b).

In this experiment, SAXS is used for the determination of the structure of AOT/ $\mathrm{H}_{2} \mathrm{O} /$ Decane microemulsions at $293.15 \mathrm{~K}$. The SAXS methods are useful for studies the structure of reverse micelles. This is because the scattering of X-rays of wavelengths of a few angstroms through small angles provides a $Q$ range (as defined by Equation 2) which is particularly appropriate for the determination of both 


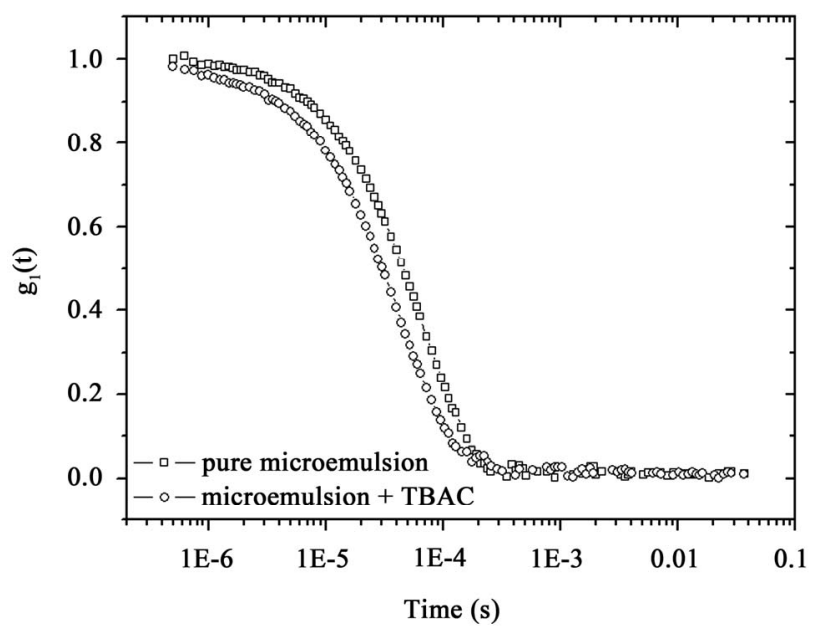

(a)

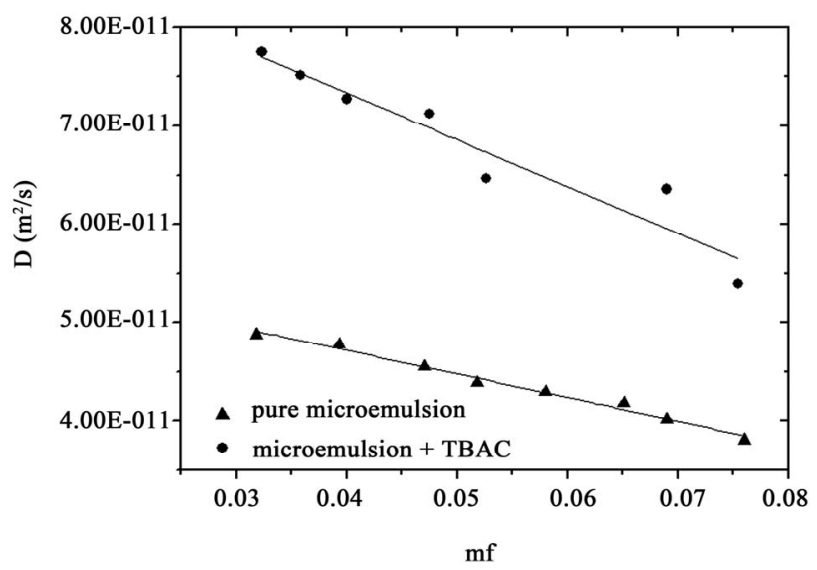

(b)

Figure 1. (a) Autocorrelation function before and after mixture of TBAC with AOT/H2O/Decane microemulsion (b) The collective diffusion coefficient as a function of mass fraction (mf) for water to AOT and Decane with $X=6.7$ (circle) and $\mathrm{X}=6.7,[\mathrm{TBAC}] /[\mathrm{AOT}]=0.049 \mathrm{molar} / \mathrm{l}$ (Down triangle) at the temperature $293.15 \mathrm{~K}$.

the size of discrete reverse micelles and their interactions. From the angular dependence of the scattered X-ray intensity $I(Q)$ we have

where

$$
I(Q)=S(Q) \cdot F(Q)
$$

$$
Q=\left(\frac{4 \pi n}{\lambda}\right) \cdot \sin \left(\frac{\theta}{2}\right)
$$

$Q$ is the wavelength and $\theta$ is the scattering angle. $F(Q)$ is referred to the form factor, which gives information on the dimensions of an individual reverse micelle, and $S(Q)$ is the structure factor, which provides information on inter-micelle interaction. We will consider the nature of the interactions between micelles. Information on interactions can be obtained from structure factor. The scattered intensity as a function of $Q$ from samples presented in the Figure 2, which the lines are fits to a power law,

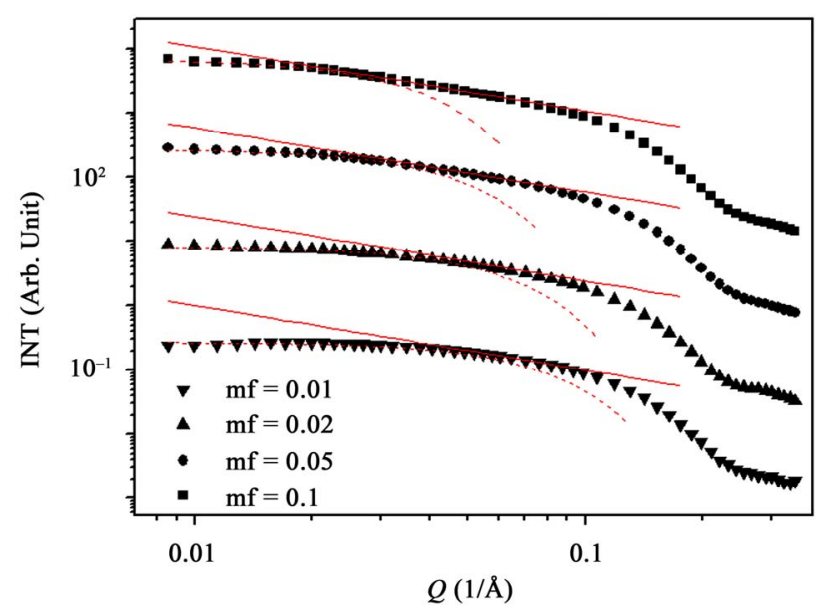

(a)

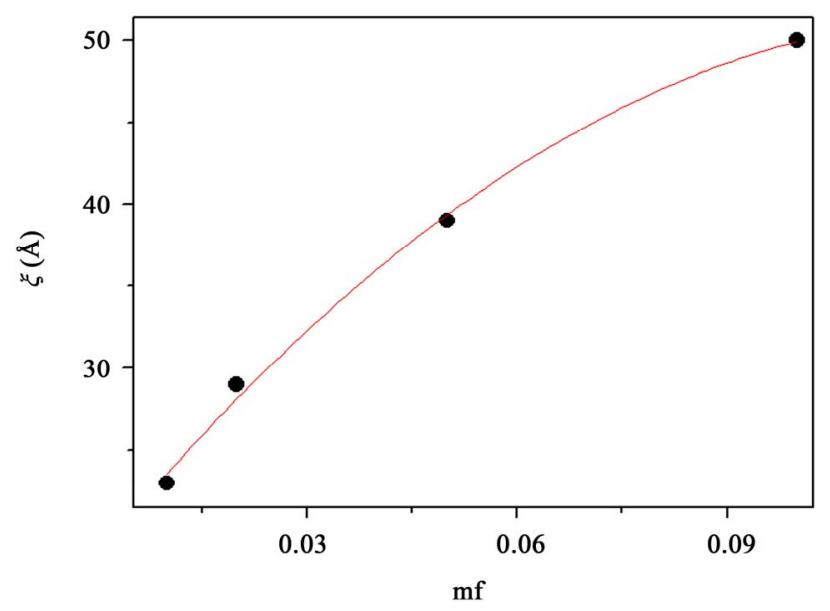

(b)

Figure 2. (a) SAXS intensity $\mathrm{I}(Q)$ of a AOT/H2O/Decane microemulsion with $X=6.7$ and different droplet mass fraction $(\mathrm{mf}=0.01,0.2,0.05,0.1)$. The red line is the $\mathrm{I}(Q) \approx Q^{-1}$ that shown cylinder behavior of the SAXS experiments and dot line at low $Q$ is the fit of Guinier's law, $\mathrm{I}(Q) \approx \exp (-(Q \xi)$ 2/3). (b) The correlation length $\xi(\AA)$ as a function of droplet mass fraction for AOT/H2O/Decane microemulsion with $X$ $=6.7$. The red line is fit with polynomial function.

$I(Q) \approx Q^{-1}$, with properties of a cylindrical object. At small $Q$, the scattering is only sensitive to the overall dimension of the scattering particles, and we expect from Guinier's law, $I(q) \approx \exp \left(-(Q \xi)^{2} / 3\right)$, where $\xi$ is correlation length. The microemulsions with molar ration 6.7 and different droplet mass fractions were collected for these experiments. The SAXS data is measured from the high concentration sample (mass fraction 0.1 ) to the dilute sample. Figure 2 shows the small angle X-ray scattering intensities for $\mathrm{AOT} / \mathrm{H}_{2} \mathrm{O} /$ Decane as a function of $Q$ at the molar ratio 6.7 and different mass fraction.

Figure 2, depict that intensity as a function of $Q$ at the high droplet mass fraction $(\mathrm{mf}=0.1)$ and $Q$ range of 0.02 $<Q(1 / \AA)<0.1$ has linear behavior. At low $Q$, we used Guinier's law, $\mathrm{I}(Q) \approx \exp (-(Q \xi) 2 / 3)$, that results pre- 
sented in the Figure 3. Our results show, the correlation length $(\xi)$ change from $23 \AA$ to $50 \AA$ with the increase of droplet mass fraction (Figure 3). The most important observation from Figure $\mathbf{2}$ is increasing of the correlation length with the increase of droplet mass fraction. We also study the effect of adding TBAC to the AOT/ $\mathrm{H}_{2} \mathrm{O} /$ Decane microemulsion with SAXS experiment. The correlation length change from $50 \AA$ to $30 \AA$ with adds TBAC to the $\mathrm{AOT} / \mathrm{H}_{2} \mathrm{O} /$ Decane microemulsion, Figure 3.

\section{Discussion}

The dynamical property of the AOT/ $\mathrm{H}_{2} \mathrm{O} /$ Decane microemulsions with and without TBAC at $\mathrm{L}_{2}$ phase is investigated by DLS technique in both types of sample we realized a single relaxation process. From analyzing of the light scattering experiments, we found the collective diffusion coefficient of the system decreases with increasing droplet mass fraction, which is in contrary with the $\mathrm{C}_{12} \mathrm{E}_{5} /$ $\mathrm{H}_{2} \mathrm{O}$ /Decane microemulsion behavior. In $\mathrm{C}_{12} \mathrm{E}_{5}$ microemulsion at low mass fraction and $\mathrm{L}_{1}$ Phase the collective diffusion coefficient as a function of the mass fraction has positive slop that means an attractive interaction between droplets, [20]. Elsewhere, shown that the size and polydispersity of $\mathrm{C}_{12} \mathrm{E}_{5} / \mathrm{H}_{2} \mathrm{O} /$ Decane microemulsion is constant with change of droplet mass fraction, [16-19], and collective diffusion coefficient as function of the mass fraction in the $\mathrm{C}_{12} \mathrm{E}_{5} / \mathrm{H}_{2} \mathrm{O} /$ Decane microemulsion only depends to the inter-particle interaction, and it comes from charge effect of droplets. But in the case of the AOT microemulsion, the SAXS experiment is shown the size of the

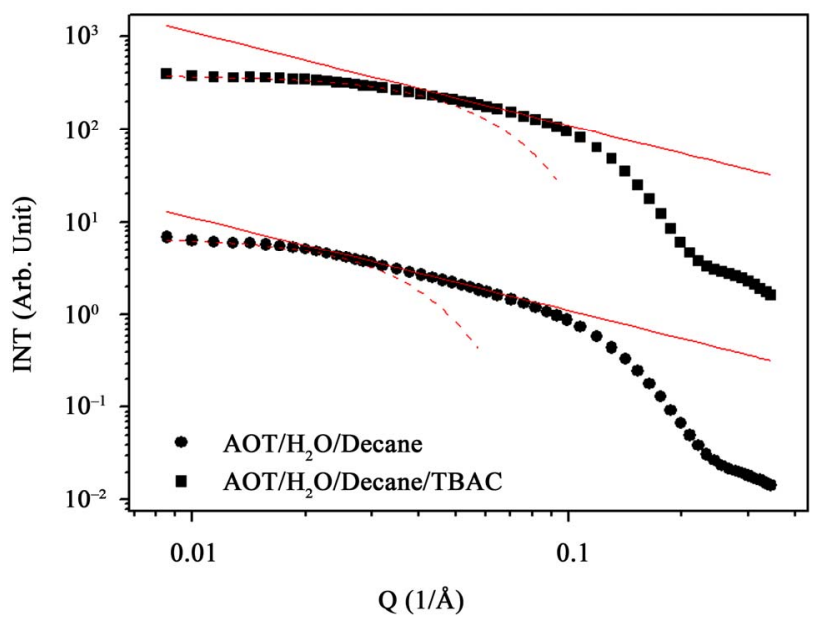

Figure 3. SAXS intensity $\mathrm{I}(Q)$ of a AOT/H2O/Decane microemulsion (circle points) with $X=6.7$ and droplet mass fraction $(\mathrm{mf}=0.1)$ and AOT/H2O/Decane/TBAC microemulsion (cubic points) with $X=6.7$ at constant droplet mass fraction $(\mathrm{mf}=0.1)$ and $[$ TBAC] $/[$ AOT $]=0.045 \mathrm{molar} / \mathrm{l}$. The red line is the line with slop $(-1)$ that shown cylinder behavior of the SAXS experiments and dot line at low $Q$ is the fit of Guinier's law, $\mathrm{I}(Q) \approx \exp (-(Q \xi) 2 / 3)$ and $\xi$ change from $50 \AA$ to $30 \AA$ with add TBAC. droplets changes from $23 \AA$ to $50 \AA$ by changing the oil concentration. In the AOT microemulsions with increase of mass fraction viscosity and size increasing and from Stokes-Einstein relation diffusion should decrease. Our results show that in the AOT microemulsion size effect and in $\mathrm{C}_{12} \mathrm{E}_{5}$ microemulsion charge effect can describe the behavior of collective diffusion coefficient. Our results show, TBAC concentration $(\mathrm{Y}=0.049 \mathrm{molar} / \mathrm{l})$ can change the collective diffusion coefficient of AOT microemulsion, Figure 1. From the SAXS measurement we obtained detailed information about the variation of the correlation length $\xi$ from $50 \AA$ to $30 \AA$ with increase of the TBAC concentration that it can describe of increase of $\mathrm{D}_{\mathrm{c}}$ with add TBAC to the AOT microemulsions.

\section{Conclusions}

The collective diffusion coefficient has been studied on AOT $/ \mathrm{H}_{2} \mathrm{O} /$ decane microemulsions shown a negative slop as a function of droplet mass fraction at the low mass fraction. Addition of TBAC to the $\mathrm{AOT} / \mathrm{H}_{2} \mathrm{O} /$ decane microemulsion changes the collective diffusion parameters. Systematic small-angle $\mathrm{X}$-ray scattering measurements have been conducted and analyzed. They reveal that these microemulsions show a change of the correlation length at very low molar ratios. In addition, the structures grow in length with decreasing concentration of oil or with increaseing of the droplet mass fraction. The change in the correlation length of droplets can describe the negative slop of the collective diffusion coefficient as function of the mass fraction and also increasing in of $D_{c}$ with the increase in TBAC concentration.

\section{Acknowledgments}

This work was supported by the University of Sistan and Baluchestan and SFB-569 (DFG), Research Collaborative Center of the Institut für Experimentelle Physik, Univer-sität Ulm.

\section{REFERENCES}

[1] Peter Schurtenberger, "Static and Dynamic Properties of Micelles, Microemulsions and Vesicles," Colloid \& Interface Science, Vol. 1, No. 6, 1996, pp. 773-778.

[2] Y. Hattori, H. Ushiki, L. Courbin and P. Panizza, "Slow Relaxation Mode in Concentrated Oil-In-Water Microemulsions Consisting of Repulsive Droplets," Physical Review E, Vol. 75, No. 2, 2007, pp. 021504-021511. doi:10.1103/PhysRevE.75.021504

[3] J. Gapinski, A. Patkowski, A. J. Banchio, P. Holmqvist, G. Meier, M. P. Lettinga, and G. Nägele, "Collective Diffusion in Charge-Stabilized Suspensions: Concentration and Salt Effects," Journal of Chemical Physics, Vol. 126, No. 10, 2007, pp. 104905-104917. doi:10.1063/1.2538891

[4] G. D. J. Phillies, G. B. Benedek and N. A. Mazer, "Diffu- 
sion in Protein Solutions at High Concentrations: A. Study by Quasielastic Light Scallering Spectroscopy," Journal of Chemical Physics, Vol. 65, No. 5, 1976, p. 1883. doi: $10.1063 / 1.433282$

[5] P. Prinsen and T. Odijk, "Collective Diffusion Coefficient of Proteins with Hydrodynamic, Electrostatic, and Adhesive Interactions," Journal of Chemical Physics, Vol. 127, No. 11, 2007, pp. 115102-115109.

[6] A. P. Minton and P. D. Ross, "Concentration Dependence of the Diffusion Coefficient of Hemoglobin," The Journal of Physical Chemistry, Vol. 82, No. 17, 1978, pp. 19341938. doi:10.1021/j100506a016

[7] N. Meechai, A. M. Jamieson and J. Blackwell, "Translational Diffusion Coefficients of Bovine Serum Albumin in Aqueous Solution at High Ionic Strength," Journal of Colloid and Interface Science, Vol. 218, No. 1, 1999, pp. 167-175.

[8] M. R. Wattenbarger, V. A. Bloomfield, Z. Bu and P. S. Russo, "Tracer Diffusion of Proteins in DNA Solutions," Macromolecules, Vol. 25, No. 20, 1992, pp. 5263-5265. doi:10.1021/ma00046a024

[9] J. B. Bishop, W. J. Fredericks, S. B. Howard and T. Sawada, "Dynamic Light Scattering Analysis of Solutions from which Lysozyme Crystals Grow," Journal of Crystal Growth, Vol. 122, No. 1-4, 1992, pp. 41-49. doi:10.1016/0022-0248(92)90224-7

[10] J. Sjöblom, K. Rosenqvist and P. Stenius, "Reversed Micellar Solutions in the System Sodium Octanoate/Decanol/Water: Model Calculations and Dynamic Light Scattering Measurements," Colloid \& Polymer Science, Vol. 260, No. 1, 1982, pp. 82-88. doi:10.1007/BF01447679

[11] A. Dobek, "Measurements of Light Scattering Changes Induced by a Strong Optical Field in Solutions of tRNA," Journal of Polymer Science: Polymer Physics Edition, Vol. 19, No. 2, 1981, pp. 273-280. doi:10.1002/pol.1981.180190208

[12] F. Mallamace, N. Micali and C. Vasi, "Viscoelastic Properties of Charged Colloids, Polystyrene, and Silica-Water Suspensions," Physical Review A, Vol. 42, No. 12, 1990, pp. 7304-7311. doi:10.1103/PhysRevA.42.7304

[13] Dale W. Schaefer, "Colloidal Suspensions as Soft Core Liquids," Journal of Chemical Physics, Vol. 66, No. 9, 1977, pp. 3980-3985. doi:10.1063/1.434449

[14] P. N. Segrè, S. P. Meeker, P. N. Pusey and W. C. K. Poon, "Viscosity and Structural Relaxation in Suspensions of Hard-Sphere Colloids," Physical Review Letters, Vol. 75, No. 5, 1995, pp. 958-961. doi:10.1103/PhysRevLett.75.958
[15] T. Nose and B. Chu, "Static and Dynamical Properties of Polystyrene in Trans-Decalin. 1. NBS 705 Standard Near $\theta$ Conditions," Macromolecules, Vol. 12, No. 4, 1979, pp 590-599. doi:10.1021/ma60070a010

[16] M. Nayeri, M. Zackrisson and J. Bergenholtz, "Scattering Functions of Core-Shell-Structured Hard Spheres with Schulz-Distributed Radii," The Journal of Physical Chemistry B, Vol. 113, No. 24, 2009, pp. 8296-8302. doi:10.1021/jp811482w

[17] H. Bagger-Jörgensen, L. Coppola, K. Thuresson, U. Olsson and K. Mortensen, "Phase Behavior, Microstructure, and Dynamics in a Nonionic Microemulsion on Addition of Hydrophobically End-Capped Poly (Ethylene Oxide)," Langmuir, Vol. 13, No. 16, 1997, pp. 4204-4218. doi:10.1021/1a9620541

[18] E. Feitosa, W. Brown and Per Hansson, "Effect of Temperature on the Interaction between the Nonionic Surfactant C12E5 and Poly (Ethylene Oxide) Investigated by Dynamic Light Scattering and Fluorescence Methods," Macromolecules, Vol. 29, No. 21, 1996, pp. 6837-6846. doi:10.1021/ma9600771

[19] M. Schwab and B. Stühn, "Relaxation Phenomena and Development of Structure in a Physically Crosslinked Nonionic Microemulsion Studied by Photon Correlation Spectroscopy and Small Angle X-Ray Scattering," Journal of Chemical Physics, Vol. 112, No. 14, 2000, pp. 6461-6472. doi:10.1063/1.481207

[20] M. Zackrisson, R. Andersson and J. Bergenholtz, "Depletion Interactions in Model Microemulsions," Langmuir, Vol. 20, No. 8, 2004, pp. 3080-3089. doi:10.1021/la036132y

[21] M. Kotlarchyk, S.-H. Chen, J. S. Huang and M. W. Kim, "Structure of Three-Component Microemulsions in the Critical Region Determined by Small-Angle Neutron Scattering," Physical Review A, Vol. 29, No. 4, 1984, pp. 2054-2069. doi:10.1103/PhysRevA.29.2054

[22] T. Blochowicz, C. Gögelein, T. Spehr, M. Müller and B. Stühn, "Polymer-Induced Transient Networks in Water-In-Oil Microemulsions Studied by Small-Angle X-Ray and Dynamic Light Scattering," Physical Review E, Vol. 76, No. 4, 2007, pp. 041505-041514.

[23] J. Bergenholtz, A. Romagnoli and N. J. Wagner, "Viscosity, Microstructure, and Interparticle Potential of AOT/ H2O/n-Decane Inverse Microemulsions," Langmuir, Vol. 11, No. 5, 1995, pp. 1559-1570.

[24] U. Batra, W. B. Russel and J. S. Huang, "Viscosity Anomaly and Charge Fluctuations in Dilute AOT Microemulsions with $\mathrm{X}<20$," Langmuir, Vol. 15, No. 11, 1999, pp. 3718-3725. 